\title{
Equalization control strategy of medium voltage direct hanging energy storage system
}

\author{
Guanjun $\mathrm{Li}^{*}$, Haoyuan Li, Fubao Wu, Shi Yin \\ National Key Laboratory on Operation and Control of Renewable Energy and Energy Storage \\ China Electric Power Research Institute Co., Ltd, Nanjing, Jiangsu, 210003, China
}

\begin{abstract}
The medium voltage direct hanging energy storage system has been widely used in high capacity applications, which gets rid of power frequency transformer and is of high conversion efficiency. This paper introduces the medium voltage direct hanging energy storage system topology scheme. In order to improve the battery life and capacity utilization, the in-phase and inter-phase battery equalization control strategies are studied. Finally, the simulation results on medium voltage direct hanging energy storage system prove the correctness and effectiveness of the equalization control strategy.
\end{abstract}

\section{Introduction}

Renewable energy power generation is developing rapidly, among which wind power generation and photovoltaic power generation are the most representative [1]. However, the intermittent and fluctuating characteristics of renewable energy output such as wind farms and photovoltaic power stations have brought a significant impact on the stable operation of power system and power quality control [2]. At present, energy storage technology has become an important part of power system operation. Energy storage technology can effectively solve the uncontrollability and nonschedulable of renewable energy generation, so as to improve the power system's ability to absorb renewable energy [3].

The energy storage system is composed of energy storage unit and power converter system (PCS) [4]. Due to the limitation of the tolerance of single transistor devices, the traditional high-capacity energy storage system mostly adopts low-voltage parallel structure with isolation transformer to realize grid connection. Due to the isolation transformer, this kind of energy storage system is of large volume, large floor area, large loss and low overall efficiency. Moreover, due to the use of traditional power conversion circuit, the switching frequency is not high, and the output waveform contains much harmonics, which reduces the power quality. In the medium voltage direct hanging energy storage system, multiple DC units are cascaded to realize the voltage rise, so as to meet the grid connection conditions, and the isolation transformer is omitted. Therefore, compared with the traditional system, the volume of the whole system is much reduced, the loss is further reduced, and the overall efficiency is improved. Due to the multi-level structure and carrier phase-shift modulation method, the equivalent switching frequency is increased to $2 \mathrm{~N}$ times of switching frequency, the harmonic content of output voltage and current waveform is greatly reduced, and the waveform tends to be sine wave, and the power quality is improved [5].

However, the DC side of the medium voltage direct hanging energy storage system is independent of each other, and there are parallel loss and pulse delay in the process of energy transmission, which leads to the energy imbalance of energy storage unit. The equalization control of battery state of charge (SOC) is important, which ensures battery life and improves battery capacity utilization [6]. If the SOC imbalance cannot be improved in time, the output performance and battery life of the energy storage device will be affected. In order to solve this problem, this paper studies the inphase and inter-phase equalization control strategies. The topology of medium voltage direct hanging energy storage system is introduced, and simulation results of in-phase and inter-phase equalization control strategy are given.

\section{Topology of medium voltage direct hanging energy storage system}

At present, the energy storage system access scheme which is widely used is low-voltage parallel collection boost energy storage access technology, which meets the power and capacity requirements of energy storage system. Based on the classic low-voltage energy storage system DC/AC, a public AC bus is built in the lowvoltage side to collect and connect to the power grid. In order to improve the capacity of single energy storage unit and improve the power quality, the application of three-level converter in battery energy storage has been

liguanjun@epri.sgcc.com.cn 
studied. Under the same device conditions, three level energy storage converter can obtain higher output voltage and higher capacity than two-level energy storage converter, which is suitable for the occasions with large capacity demand of single machine.

Fig. 1 shows the medium voltage direct hanging energy storage system based on the traditional H-bridge cascade topology. The advantage is that the transformer is not required to be directly connected to the power grid, so as to meet the demand of single machine large capacity of energy storage system. For dispatching command response, the dispatching response consistency is good and the response rate is fast. The power conversion efficiency of the system can be improved, because there is no need for step-up transformer. Furthermore, the system has high fault tolerance operation ability, and can set redundant submodules.

Fig. 2 shows the medium voltage direct connected energy storage system based on MMC topology. The topology is a three-phase six-leg symmetrical structure, every phase is divided into two arms, which is composed of several power units and inductors in series. MMC has a highly modular topology, which can expand the power capacity by changing the number of sub modules in every arm. The bridge arm reactor can suppress the second harmonic circulating current and buffer the impact of fault current. Compared with H-bridge cascaded direct connected energy storage system, the biggest advantage is that $\mathrm{ABC}$ three-phase constructs a common DC bus. If there is a demand for medium and high-voltage DC power grid access to energy storage system, MMC based energy storage access technology is more applicable. However it is less used in the field of energy storage system, due to its own topology limitation, the number of sub modules is too large and the cost is high.

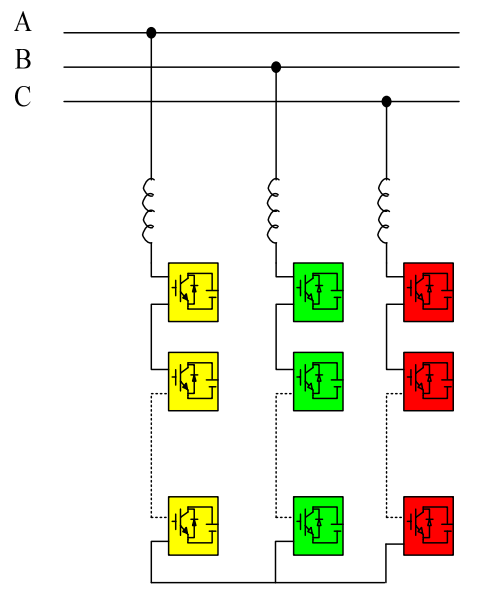

Figure 1. Medium voltage direct hanging energy storage system based on traditional H-bridge cascade topology.

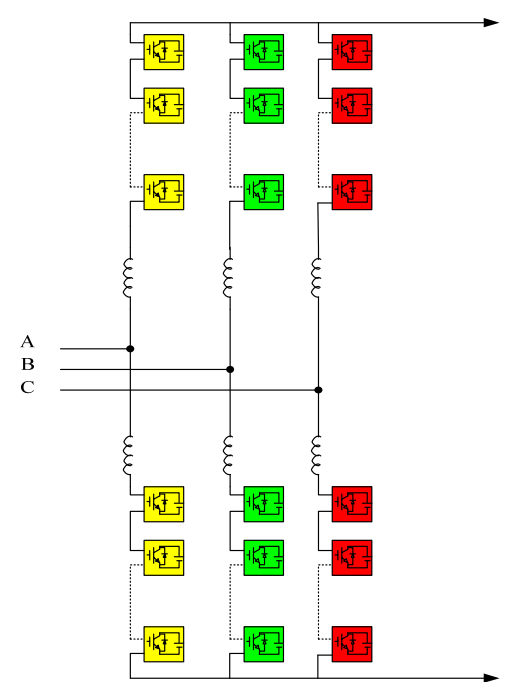

Figure 2. Medium voltage direct hanging energy storage system based on MMC topology.

\section{Battery SOC equalization control of medium voltage direct hanging energy storage system}

The equalization control of battery state of charge (SOC) is very important, which ensures battery life and improves battery capacity utilization. SOC equalization control is divided into in-phase SOC equalization control and inter-phase SOC equalization control.

\subsection{Analysis of SOC equalization control principle of inter-phase battery cluster}

The purpose of inter-phase SOC equalization control is to ensure the average value of three-phase SOC consistent. As shown in Fig. 3, inter-phase SOC equalization control is realized by injecting zero sequence voltage to change the inter-phase power distribution of the system. Since the system is Y-type three-phase three wire system, there is no zero sequence current path, so the additional zero sequence voltage will not affect the total output power of the energy storage system. 


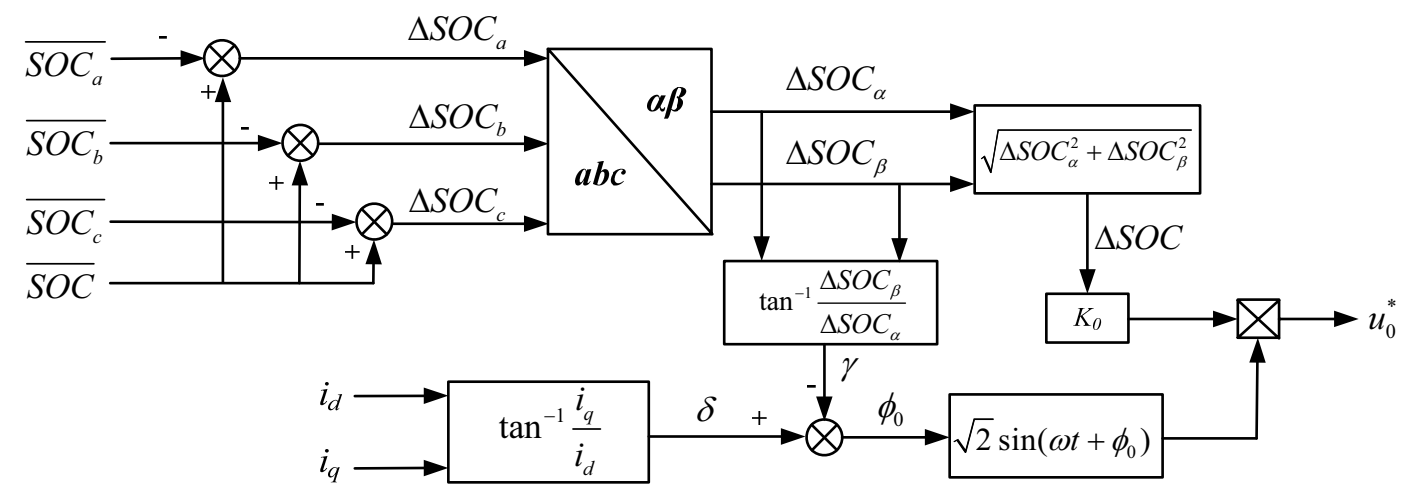

Figure 3. Control block diagram of inter-phase SOC equalization.

$\overline{S O C}_{a}, \overline{S O C}_{b}, \overline{S O C}_{c}$ are the average SOC value of three phases, respectively, $\overline{S O C}$ represents the overall average SOC value. The difference among $\overline{S O C}_{a}$, $\overline{S O C}_{b}, \overline{S O C}_{c}$ are transformed into $\triangle S O C_{\alpha}$ and $\triangle S O C_{\beta}$. Then the injected zero sequence voltage can be expressed as

$$
u_{0}^{*}=\sqrt{2} K_{0} \cdot \Delta S O C \cdot \sin (\omega t+\delta-\gamma)
$$

where $K_{0}$ represents proportional coefficient, while other parameters can be calculated as follows

$$
\left\{\begin{array}{c}
\triangle S O C=\sqrt{\triangle S O C_{\alpha}^{2}+\triangle S O C_{\beta}^{2}} \\
\delta=\tan ^{-1} \frac{i_{q}}{i_{d}} \\
\gamma=\tan ^{-1} \frac{\Delta S O C_{\beta}}{\Delta S O C_{\alpha}}
\end{array}\right.
$$

\subsection{Analysis of SOC equalization control principle of in-phase link battery}

In-phase SOC equalization control is to ensure that the SOC values of each module in one phase are consistent. The output power of each power unit of each link should be positively correlated with the corresponding battery cell SOC. Since the current flowing through one phase power unit is the same, the realization of in-phase SOC equalization control can be realized by adding corresponding fundamental voltage components of each unit. The calculation block diagram of in-phase SOC equalization control component is shown in Fig. 4, where $K_{1}$ is the proportional coefficient.

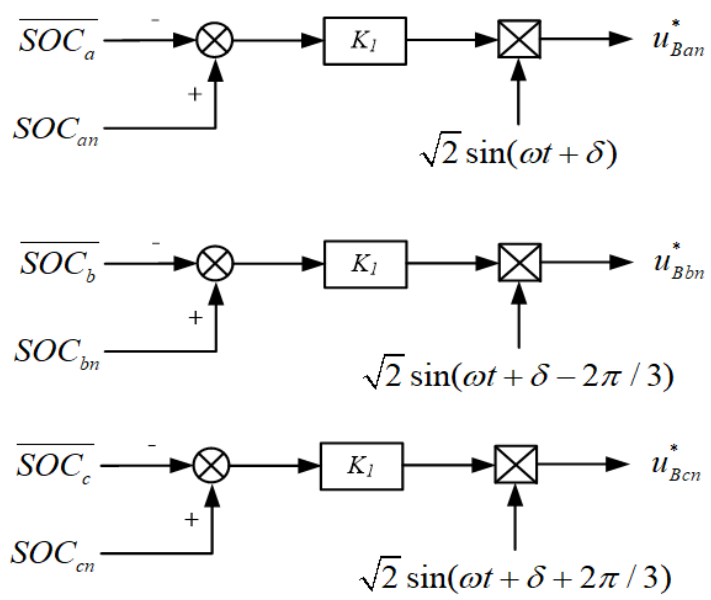

Figure 4. Control block diagram of phase SOC equalization.

Combined with in-phase and inter-phase SOC equalization control strategy, the overall voltage command is obtained by three phase voltage references $u_{a}^{*}, u_{b}{ }^{*}, u_{c}{ }^{*}$, adding the voltage regulation after battery SOC equalization control.

\section{Simulation results of SOC equalization control}

\subsection{SOC equalization simulation in system discharge mode}


The system is worked in the rated state, and the power is set as $5 \mathrm{MW}$. The voltage value of 42 units of phase $\mathrm{A}$ is set as $950 \mathrm{v}, 42$ units of phase B is set as $850 \mathrm{~V}$, and 42 units of phase $\mathrm{C}$ is set as $750 \mathrm{~V}$. The system turns on the function of battery inter-phase equalization after $1 \mathrm{~s}$. The output current and output power of the system are not affected when the inter-phase equalization control function is turned on. Fig. 5 shows the battery current of the first power unit of the system A, B and C. The output power of the three phases is consistent before the interphase equalization control is turned on, the SOC of phase $\mathrm{A}$ is the largest, so the battery current of phase A is the smallest. After turning on the inter-phase equalization control, it is necessary to increase the output power of phase A power unit and reduce the output power of phase $\mathrm{C}$ power unit, in order to make the three-phase SOC consistent.

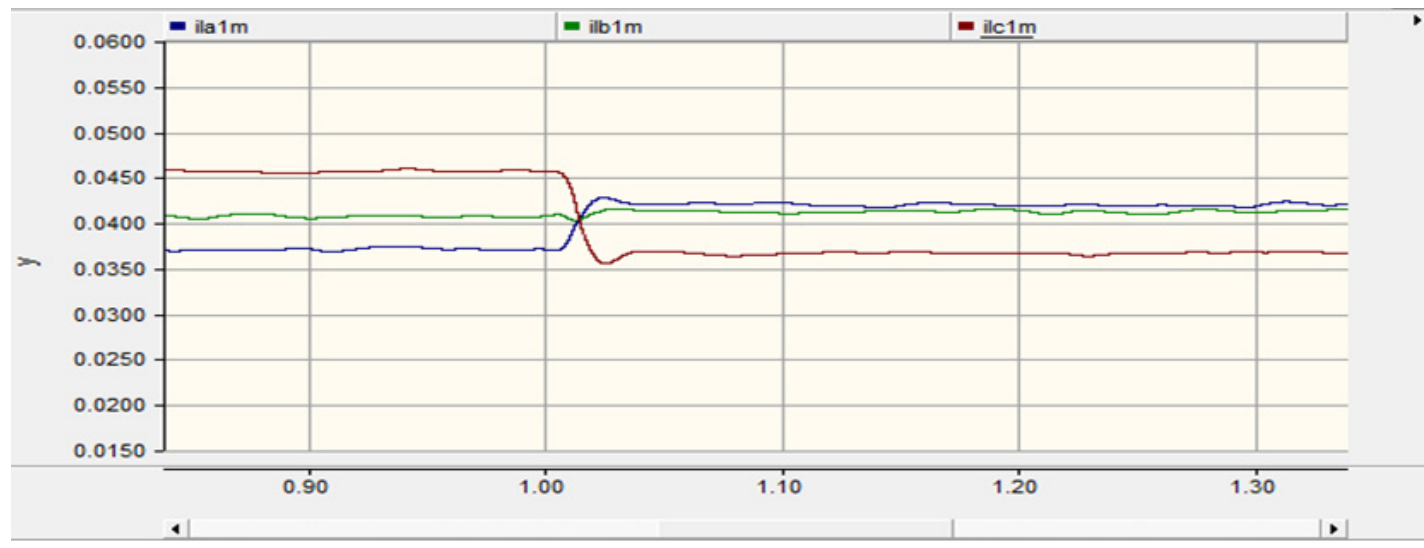

Figure 5. Effective value of three-phase battery current.

Fig. 6 shows the output power of the first power unit of three phases A, B and C. The output power of threephase power units is consistent before the inter-phase equalization control of SOC is turned on. After switching on the phase to phase equalization control of SOC, the output power of phase A unit increases and that of phase $\mathrm{C}$ decreases.

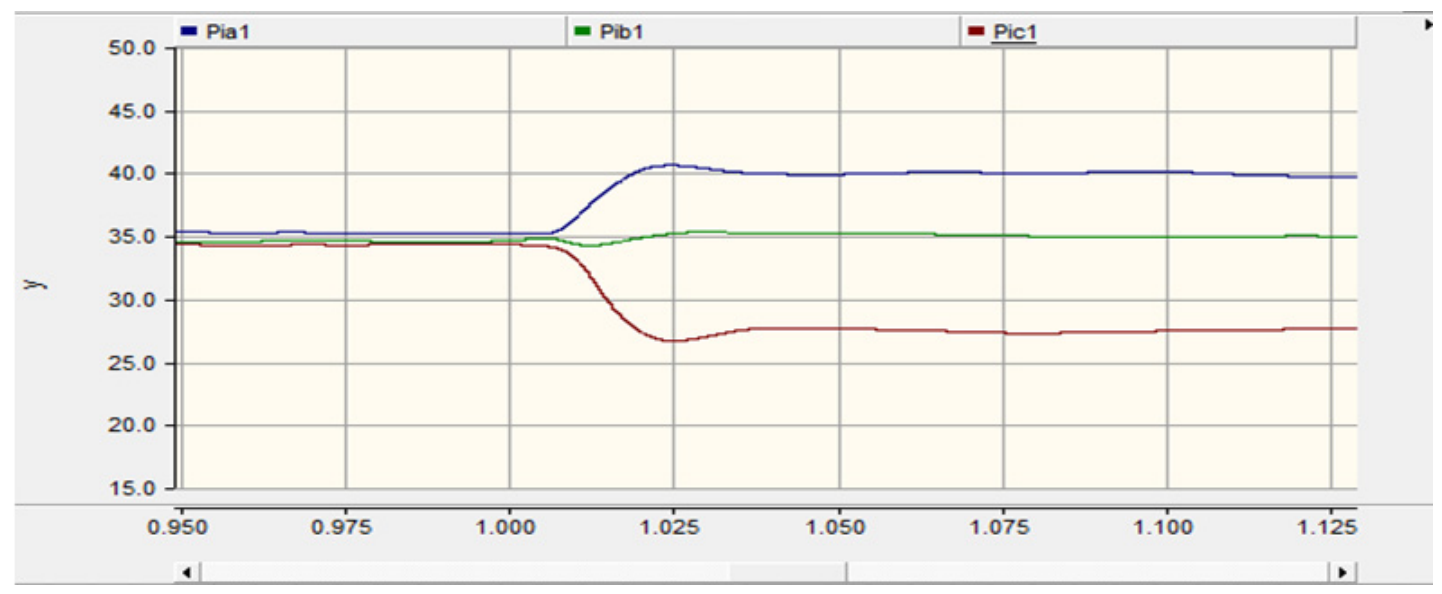

Figure 6. Output power of three-phase power unit (kw).

\subsection{Simulation of SOC equalization control for in-phase link battery}

The system is worked in the rated state, and the power is set as $5 \mathrm{MW}$. The voltage value of the first unit of phase $A$ is $950 \mathrm{v}$, the voltage value of the second unit of phase $A$ is $750 \mathrm{~V}$, and the voltage value of other units is $850 \mathrm{~V}$. Besides the voltage value of all units of phase $\mathrm{B}$ and phase $\mathrm{C}$ is $850 \mathrm{~V}$. The function of in-phase equalization of battery is turned on after $0.5 \mathrm{~s}$. The output current and output power of the system are not affected by the function of in-phase equalization control. Fig. 7 shows the battery current of the first, second and third power units of phase A. It is shown that before the in-phase equalization control is turned on, the SOC of the first unit of phase A is the largest, so the battery current of the first unit is the smallest, under the condition that the output power of the three phases is the same. Meanwhile the SOC of the second unit is the smallest, thus the battery current of the second unit is the largest. After the in-phase equalization control is turned on, the battery current of the first unit becomes larger, as the increase of the output power. While the battery current of the second cell becomes smaller and the output current becomes smaller. 


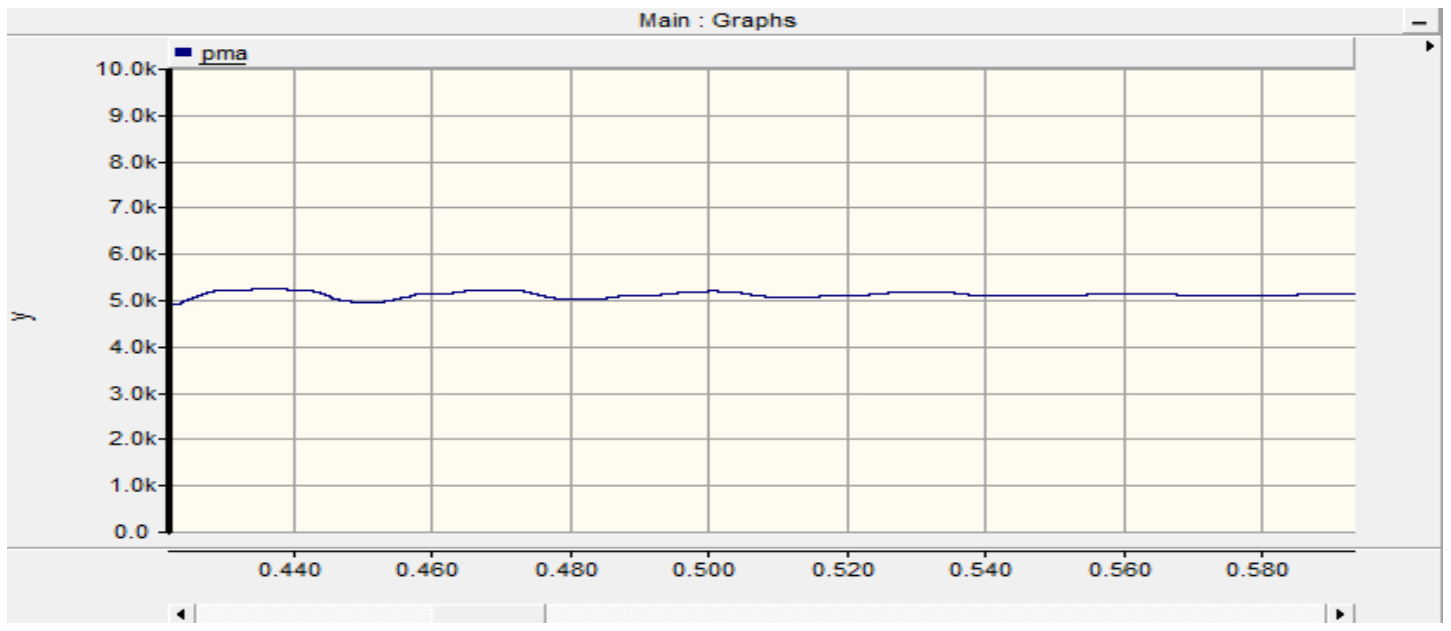

Figure 7. Effective value of three-phase battery current.

\subsection{Equalization control in charging mode}

The system is worked in the rated state, and the power is set as $5 \mathrm{MW}$. The voltage value of the first unit of phase $A$ is $950 \mathrm{v}$, the voltage value of the second unit of phase $\mathrm{A}$ is $750 \mathrm{~V}$, and the voltage value of other units is $850 \mathrm{~V}$. Besides the voltage value of all units of phase $\mathrm{B}$ and phase $\mathrm{C}$ is $850 \mathrm{~V}$. The function of in-phase equalization of battery is turned on after $0.5 \mathrm{~s}$. The output current and output power of the system are not affected by the function of in-phase equalization control. Fig. 8 shows the battery current of the first, second and third power units of phase A. Before the in-phase equalization control is turned on, the SOC of the first unit of phase A is the largest, so the battery current of the first unit is the smallest, because that the output power of the three phases is the same. Furthermore, the SOC of the second unit is the smallest, thus the battery current of the second unit is the largest. After the in-phase equalization control is turned on, the active power absorbed by the first unit is increased as the power decreases, and the battery becomes smaller. Besides, the active power absorbed by the second unit increases, and the battery becomes larger.

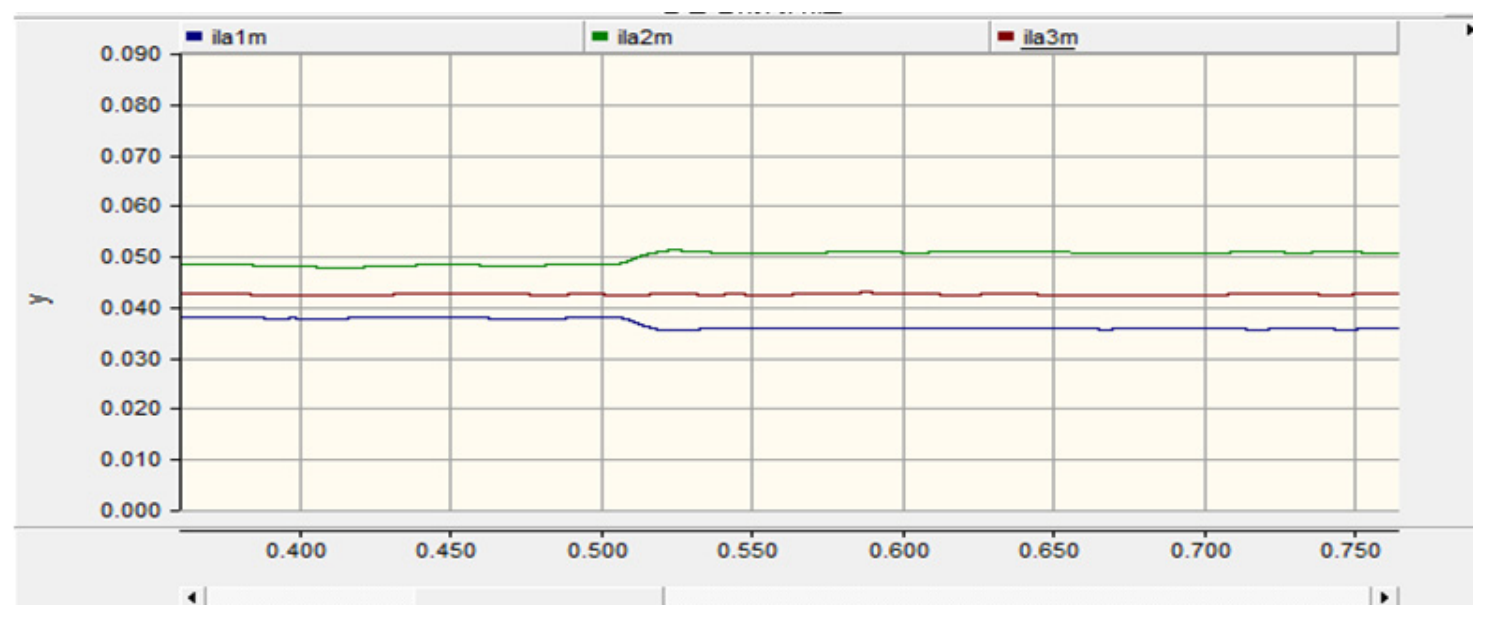

Figure 8. Effective value of battery current of the 1st, 2nd and 3rd units of phase a (kA).

\section{Conclusion}

This paper has studied the topology of medium voltage direct hanging energy storage system, as well as the inphase and inter-phase SOC equalization control strategies. It is shown that the medium voltage direct hanging energy storage system itself has the ability of SOC equalization, which can balance the SOC of inphase and inter-phase battery cells. Therefore, the work of BMS of each battery cell in the power converter system can be simplified and the cost of BMS can be greatly reduced.

\section{Acknowledgment}

This work was supported by the State Grid Corporation Headquarters Science and Technology Project, i.e., Research and Application of Active Support Technology for Large Scale New Energy Power Station Based on Energy Storage. 


\section{References}

1. Mei N., Yuan B., Li T., et al. (2018) Study on control strategy of bipolarise station connected to islanded renewable power plant [J]. Power System Technology, 42: 3575-3582.

2. Zhang Z., Li L., Ding S.Y., et al. (2019) Modeling and operation strategy research on flexible wind farm grid-connection auxiliary system [J]. Power System Technology, 43: 1221-1226.

3. Li J.L., Ma H.M., Yuan X.D., et a1. (2017) Overview on key applied technologies of large-scale distributed energy storage [J]. Power System Technology, 41: 3365-3375.

4. Zheng Z., Wang Y.S., Zhang G.P., et al. (2020) Study on energy balance control strategy of energy storage unit in chain energy storage system [J]. Power System Technology, 44: 1673-1683.

5. Ling Z.B., Cao Y., Ma Q.D., et al. (2014) Design of $2 \mathrm{MW} / 10 \mathrm{kV}$ cascaded power conversion system[C]. In: 40th Annual Conference of the IEEE Industrial Electronics Society. Dallas, TX. pp. 4250-4255.

6. Hu W.F., Shi H.B., Li G.J., et al. (2020) Power distribution control for cascaded multilevel inverter with hybrid energy sources based on initial operation point selection [J]. Power System Technology, 44: 1639-1651. 